\title{
SALUD MENTAL
}

\section{Riesgo de salud mental durante la formación de especialistas}

\author{
Arnaldo Jordán Alfonso', Marcos Hernán Capurro², María del Pilar \\ Fresco ${ }^{1}$, Carmen Marina Arrom Suhur ${ }^{3}$, María Auxiliadora Arrom \\ Suhurt ${ }^{3}$, Cristina Arrom Suhurt ${ }^{1,3}$
}

\begin{abstract}
Resumen
Introducción: La vida del médico residente que se encuentra en etapa de formación tiene gratificaciones como también insatisfacciones que provienen de las tareas propias del trabajo y tienen un impacto en la salud física y mental que puede devenir en trastornos afectivos de riesgo, como la depresión. Estas vivencias tienen impacto en la salud física y mental del médico y pueden devenir en trastornos que causan deterioro en la actividad social y laboral del sujeto que las padece, como los trastornos afectivos, entre ellos la depresión. Estudiantes de medicina y residentes médicos son extremadamente reacios en reportar historias de depresión durante entrevistas de selección muy competitivas. La prevalencia de depresión en esta población está estimada en un rango de 15-30\%.
\end{abstract}

Objetivo: Determinar el riesgo suicida en médicos residentes de un Hospital Escuela.

Material y Método: Estudio descriptivo con componente analítico, de corte transversal realizado de noviembre a diciembre de 2015 como trabajo final de la especialidad de Psiquiatría. Muestreo no probabilístico, por conveniencia de médicos residentes que se encontraban cumpliendo funciones académicoasistenciales al momento del estudio, aceptaron colaborar en forma voluntaria con un consentimiento informado. Se utilizó un cuestionario autoadministrado con datos sociodemográficos y la Escala de Riesgo Suicida de Plutchik. Resultados: El mayor porcentaje de la muestra, 72\% (103) se concentró en el rango de 25 a 30 años. El 48,3\% (69) de sexo femenino y 45,5\% (65) masculino. La mayoría, 62,9\% (90) se encontraba soltero/a. Del total de personas con riesgo suicida 15,3\% (20), $65 \%$ (13) fueron del sexo femenino y $35 \%$ (7) masculino. 30\% (6) fueron de la especialidad de Medicina Interna, 20\% (4) de Pediatría y 50\% restante de otras especialidades.

Conclusiones: La prevalencia riesgo suicida es mayor que en la población general.

1. Universidad Nacional de Asunción, Cátedra de Psiquiatría.

2. Centro Nacional de Control de Adicciones.

3. Centro para el Desarrollo de la Investigación Científica (CEDIC).

E-mail: marcoshcapurro@hotmail.com

DOI: 10.26885/rcei.foro.2018.148 


\section{Riesgo de salud mental durante la formación de especialistas. Alfonso et al.}

El sexo femenino y ser soltero se relacionan con mayor riesgo. Algunos autores concluyen que la tasa de suicidio para las médicas es más alta en comparación a la población en general y médicos. Otros hablan de que las médicas realizan menor número de tentativas suicidas que su contraparte en la población general, la consumación del suicidio tiene prevalencias cercanas a la de los médicos varones, por lo tanto, mucho mayor que en la población general (2,5 a 4 veces). Aunque se encontró mayor riesgo suicida en Medicina Interna y Pediatría, se necesita de mayor tamaño de muestra para correlaciones significativas. Se encontró correlación significativa entre depresión y riesgo suicida, aunque se debe comentar que no todos los consultantes que clasificaron para riesgo suicida lo hacían para depresión, lo cual nos sugiere la necesidad de control de las dos variables al tiempo de proponer el cribado. Se recomienda ampliar este estudio, realizando mediciones en por lo menos dos momentos del año académico, desestigmatizar el uso de dispositivos de salud mental, promover espacios de reflexión acerca del currículo oficial y oculto en las residencias médicas, elaborar programas de prevención, detección precoz y acompañamiento al médico residente, asegurando confidencialidad, disminuyendo así las barreras para el acercamiento de estos a esta clase de programas.

Palabras clave: riesgo suicida, médicos residentes, hospital escuela.

\section{REFERENCIAS}

López, I., Valdés, M. (2003). Manual diagnóstico y estadístico de los trastornos mentales (DSM-IV.TR). Asociación Americana de Psiquiatría. Masson, México.

Mechanic, D. (2003). Physician discontent: challenges and opportunities. Jama, 290(7), p. 941-946.

Reames, H., Dunstone, D. (1989). Professional satisfaction of physicians. Archives Of Internal Medicine,149(9), 1951-1956.

Rojas, A. (2012). The hidden curriculum in medical teaching. Revista Médica De Chile, 140(9): 1213-1217.

Shaw, D. L., Wedding, D., \& Zeldow, P. B. (2001). Suicide among medical students and physicians, special problems of medical students. In D. Wedding(Ed.), Behavior and Medicine (3rd ed., pp. 78-79). Hogrefe and Huber. 